Graham Hart, BA, PhD

Centre Director, Centre for Sexual Health and HIV Research, University College London,

London, UK

Audrey Prost, BA, PhD

Research Fellow, MRC Social and Public Health Services Unit, Glasgow, UK

References

Griffiths C, Prost A, Hart G. Sexual and reproductive ealth of South Asians in the UK: an overview. J Fam Plan Reprod Health Care 2008; 34: 251-260.

2 Department of Health. Abortion Statistics, England and Wales: 2007, Statistical Bulletin 2008/01. London, UK: Wales. 2007, Statistical Bullitir

3 Berthoud R. Teenage births to ethnic minority women. Popul Trends 2001: 104:12-17.

Department for Children, Schools and Families Department for Children, Schools and Families DCSF). Teenage Parents Next Steps. Guidance for Parents 2007. Families, 2007 .

5 Health Protection Agency. NCSP: Five Years. The Fifth Annual Report of the National Chlamydia Screening Programme 2007/2008.

Fenton KA Mercer CH, McManus S, Erens B Wellings $K$, Macdowall W Wellings $K$, Macdowall $W$, et al. Ethnic variations in . 2005: 365: 1246-1255.

\section{Missing IUS arms?}

We want to describe a couple of cases that serve to demonstrate that the hormone capsule of the Mirena ${ }^{\circledR}$ intrauterine system (IUS) can dislodge during removal thus changing its appearance, As a result, careful examination of the device is required to prevent further unnecessary investigations.

A 58-year-old woman presented to her general practitioner for removal of a Mirena IUS as it was no longer required. It had been inserted at the practice 7 years previously to provide the progestogen component of her hormone replacement therapy.

At the time of removal the cervix and the IUS threads were visualised. More traction than usual was required on the threads to remove the device. On inspection it appeared that the horizontal arms had become detached as they were not evident and the vertical main stem of the IUS had been removed with the hormone release capsule attached.

The patient was asymptomatic and was allowed home. A transvaginal ultrasound scan was performed on an outpatient basis. The scan demonstrated echogenic specks at either end of the endometrium in longitudinal section, and in cross-section specks were noted within the lateral walls of the uterus. It was queried whether these represented the arms of the IUS. The patient was then referred to the gynaecology department, for consideration for operative hysteroscopy to remove the retained arms.

A 50-year-old woman presented to the colposcopy clinic with moderate dyskaryotic smears. She had undergone two previous large loop excision of the transformation zone (LLETZ) procedures for cervical intraepithelial neoplasia (CIN) with complete excision at each. On this occasion colposcopy examination was limited because of unsatisfactory views of the squamo-columnar junction. It was decided the patient would have a further LLETZ treatment with removal and reinsertion of the Mirena IUS under general anaesthesia.

At the time of the procedure it was noted that the IUS threads were visible and the internal cervical os was tight. Again more traction than usual was required on the threads to remove the device. On inspection it was thought that the arms had become detached; the long stem of the device with the hormone release capsule present was attached to the threads. A saline hysteroscopy was therefore performed to locate the IUS arms. Good views of the entire cavity failed to demonstrate the presence of IUS pieces or perforation. The LLETZ procedure was performed and a new IUS inserted. The patient underwent an uneventful post-operative recovery.

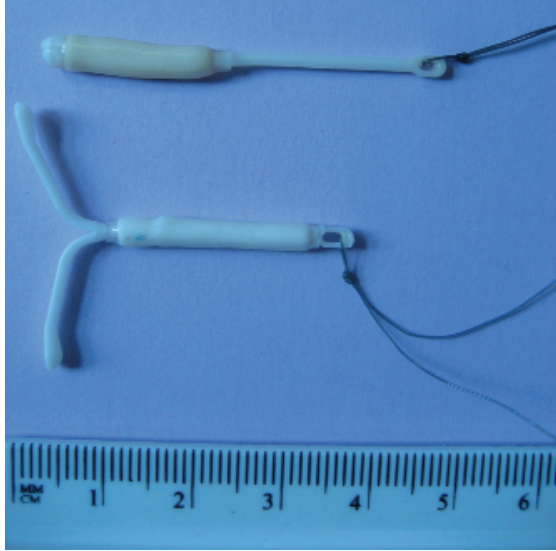

Figure 1 The intrauterine system (IUS) shown in the upper part of the photograph has been removed entirely but its appearance is atypical. The IUS in the lower part of the photograph has a normal appearance

In both cases, when the removed IUS was reexamined, it became apparent that the entire device had been removed from the uterine cavity. The arms were still attached to the main stem of the IUS. The hormone release capsule, usually situated at the base of the vertical stem, had migrated up the shaft, trapping the arms and bringing them together in the midline, making it appear as if they had been detached (Figure 1).

The majority of IUS are removed without difficulty. There are no published cases of IUS arms becoming detached. However, an intrauterine-retained hormone release capsule following IUS removal has been documented. ${ }^{1}$

The common theme in the two patients described above and Forrest et al.'s patient ${ }^{1}$ is difficult retrieval of the device requiring more traction on the threads than normal. This presumably led to the hormone capsule being dislodged, either migrating up the device and getting stuck covering the arms or becoming detached altogether. Clinicians should always check IUS devices after removal. They should also be aware that after a difficult removal the capsule can migrate and obscure the arms but the device remains complete. Knowledge of this possibility will prevent patients being subjected to unnecessary investigations and interventions to find 'missing' IUS arms and for appropriate investigations and interventions when the capsule has detached completely.

The whole of the IUS device is radio-opaque and can be located with either X-ray or ultrasound.$^{2}$ Transvaginal ultrasound is the firstline investigation because it provides the best images to help determine whether or not the IUS is correctly sited within the uterus. The vertical stem of the IUS is visualised in the sagittal plane with multiple reflective parallel planes and in the axial plane as a single echogenic focus. ${ }^{3}$ However, in the cases reported here the vertical stem was missing. Horizontal arms are rarely seen in the uterus unless it is possible to obtain a coronal view. ${ }^{3}$ In view of this difficulty abdominal X-ray would confirm whether or not the horizontal arms of the IUS were within the pelvis. This would be useful, especially prior to embarking on hysteroscopic investigation.

Emma J V Torbé, DFSRH, MRCOG

Specialist Registrar in Obstetrics and

Gynaecology, Royal Hampshire County

Hospital, Winchester, UK.

E-mail:emmatorbe@doctors.org.uk

Hillary Eddowes, MBChB, MRCOG

Associate Specialist, Royal Hampshire County Hospital, Winchester, UK

Kate Aston, $\mathrm{MBChB}$, FRCOG

Consultant, Royal Hampshire County Hospital, Winchester, UK
References

Forrest A, Amarakone I, Lord J. Retained hormone release capsule following removal of Mirena intrauterine system. Br J Obstet Gynaecol 2008; 115 130-131.

Schering. Contra-indications, warnings, etc. Mirena Insertion Instructions, June 2007.

Andrews $\mathrm{H}$, Joy V. Ultrasound in the detection of intrauterine devices. Ultrasound 2004; 12: 33-37.

Reply

We would like to take the opportunity to respond to Dr Torbe et al.'s letter.

Extremely rare, isolated case reports of hormone cylinder dislocations in the Mirena ${ }^{\circledR}$ intrauterine system (IUS) similar to the ones described by the authors have been received by the company's Pharmacovigilance and Quality Assurance Unit. The company's investigations have shown that these cases could not be attributed to a quality defect of the product. Difficult removal has been found as the underlying cause, and no further adverse effect in the Mirena user are mentioned in the majority of cases.

To make physicians aware of this extremely rare situation, and to avoid unnecessary interventions in search of 'missing' Mirena arms, the company has recently introduced the following statement into the Core Safety Information for Mirena: "After removal of Mirena ${ }^{\circledR}$, the system should be checked to be intact. During difficult removals, single cases have been reported of the hormone cylinder sliding over the horizontal arms and hiding them together inside the cylinder. This situation does not require further intervention once completeness of the IUS has been ascertained The knobs of the horizontal arms usually prevent complete detachment of the cylinder from the Tbody".

Implementation of this statement into the local product information is currently ongoing in all countries where Mirena is marketed, and it was submitted at the beginning of December 2008 to the Medicines and Healthcare products Regulatory Agency (MHRA) to be implemented in the UK.

\section{Jussi Pirjola}

Director, Quality Management Bayer Schering Pharma Oy, PO Box 415, 20101 Turku, Finland

Sarah Rybowski,

Medical Advisor, Women's Healthcare,

Bayer Public Limited Company, Strawberry Hill, Newbury, UK

E-mail: sarah.rybowski@bayerhealthcare.com

Reference

Torbé EJV, Eddowes E, Aston K. Missing IUS arms? [Letter]. J Fam Plann Reprod Health Care 2009; 35: 131.

\section{Serious morbidity with long-term} UUD retention

We have recently encountered four patients with serious intraperitoneal sepsis over an 18-month interval $(2007 / 2008)$. Each was associated with long-term retention of a copper intrauterine device (IUD), which was identified as the likely source of infection. The IUDs had been in situ for $8,15,18$ and 20 years, respectively. Three women were several years into their menopause. All four women presented as systemically unwell with a complex pelvic mass. One had ureteric obstruction at the site of the abscess, simulating gynaecological malignancy. In all cases laparotomy was technically difficult owing to the inflammatory pelvic mass adhering to bowel. Intermediate or prolonged hospitalisation resulted and, without intensive care, two of the women would probably have died.

Pelvic actinomycosis was reported in the two patients' histology. Cultures of frank pus grew Actinomyces sp. in a third. Actinomyces-like organisms (ALOs) had been reported on the last smear of the fourth woman. In 2004 she had undergone appendicectomy, which showed 
severe subserosal inflammation without mucosal inflammation leading to the conclusion that the source was elsewhere within the abdomen or pelvis. It is speculative that this episode 4 years earlier might also have resulted from the longterm presence of her IUD. Pelvic actinomycosis normally begins as subacute or chronic disease, months or years before presentation. ${ }^{1}$

The copper IUD devices marketed in the UK have licensed durations of 5,8 or 10 years. In women aged under 40 years it is recommended they are changed according to licence. If inserted after the age of 40 years they may remain in situ until 1 year after the menopause if the last period (LMP) is over the age of 50 years, or 2 years after if the LMP is under the age of 50 years. ${ }^{2}$ These recommendations are based on consensus opinion and acknowledge that insertion-related risks are minimised by reducing the frequency of IUD changes. National guidance places strong emphasis on when removal is safe from a contraceptive point of view. ${ }^{2}$ There is no clear mention of the need for removal once the contraceptive action is no longer required, or of the risks of failing to do so. The frequency with which ALOs are reported in routine smears rises in a linear fashion with the duration of use of devices. ${ }^{3}$ ALOs are more common with certain types of IUD (e.g. Multiload ${ }^{\circledR}$ ) and uncommon with the levonorgestrel intrauterine system. ${ }^{4}$ Pelvic actinomycosis is an uncommon and poorly understood condition, but has been recognised to complicate IUD use since the first report in 1973. However, Actinomycetes also normally reside in the female genital tract. ${ }^{2}$

We cannot provide any denominator data for the number of women in the catchment population with a long-term IUD, but the occurrence of a cluster of cases of serious intraperitoneal sepsis in a single hospital in a relatively short space of time is unusual. It is likely that single cases are not reported, or the association with the copper IUD overlooked, by surgeons and not fed back to those providing contraception services. When a pelvic mass or abscess, fever and other signs of infection are found in patients with a long-term IUD, pelvic actinomycosis should be considered. Awareness of this could usefully be increased among general surgeons and gynaecologists. We recommend that current guidelines be revised to include some emphasis on the importance of timely removal of an IUD, once its contraceptive properties are no longer required. Women should be made aware that long-term retention may rarely result in serious sepsis associated with pelvic abscesses and/or actinomycosis. There should be more emphasis on timely removal of an IUD early in the menopause. This is not included in existing professional guidance ${ }^{2}$ and patient information leaflets. ${ }^{5}$

Mary Pillai, FRCOG, MRCPCH

Consultant Gynaecologist, Gloucestershire Hospitals NHS Trust, Cheltenham General Hospital, Sandford Road, Cheltenham, UK. E-mail:mary.pillai@glos.nhs.uk

Maud Van de Venne, artsexamen Maastricht, MRCOG Specialist Registrar, Gloucestershire Hospitals NHS Trust, Cheltenham General Hospital, Sandford Road, Cheltenham, UK

J Shefras, MRCOG, MFSRH

Consultant in Sexual Health and Reproductive Medicine, Gloucestershire Hospitals NHS Trust, Cheltenham General Hospital, Sandford Road, Cheltenham, UK

1 Lely RJ, van Es HW. Pelvic actinomycosis in association with an intrauterine device. Radiology 2005; 236: 492-294.

2 Faculty of Sexual and Reproductive Healthcare Clinical Effectiveness Unit. FSRH Clinical Guidance (November 2007). Intrauterine contraception. http://www.ffprhc.org.uk/admin/uploads/CEUGuidance
IfrauterineContraceptionNov07.pdf [Accessed 10 August 2008

3 Chatwani A, Amin-Hanjani S. Incidence of actinomycosis associated with intrauterine devices. Reprod Med 1994; 39: 585-587.

4 Merki-Feld GS, Lebeda E, Hogg B, Keller PJ. The incidence of actinomyces-like organisms in Papanicolaou-stained smears of copperlevonorgestrel-releasing intrauterine devices. Contraception 2000; 61:365-368.

Family Planning Association (fpa). Your Guide to the IUD. London, UK: fpa, October 2007.

\section{Reply}

With regard to the four cases of serious pelvic infection described by Pillai et al., ${ }^{1}$ the Clinical Effectiveness Unit (CEU) acknowledges that long-term retention of an intrauterine device (IUD) is associated with infection and that the risk of actinomycotic pelvic abscess increases with duration of use. For this reason, Faculty of Sexual and Reproductive Healthcare (FSRH) Guidance recommends that an IUD is removed at the end of its licensed duration or when no longe required. ${ }^{2}$ In women having an IUD inserted between the age of 40 years and the menopause FSRH Guidance recommends that, based on expert opinion, the risk of infection in the 20 days following replacement of an IUD outweighs the risk of extending use until the menopause. In this situation IUD use can be continued until 1 year after the last menstrual period (LMP), or 2 years after the menopause if the LMP occurs under the age of 50 years. ${ }^{2}$

Interestingly, since being asked to respond on behalf of the CEU, a 70-year-old woman presented to my gynaecology clinic with vagina discharge and an IUD that had been in situ for 30 years. The threads of the IUD were visible and the patient had attended her GP practice after the menopause for cervical smear tests. She claimed that she had asked the practice nurse about removal of the IUD but had been reassured that it was not causing any harm.

It is not clear how many IUD users retain their IUD after the menopause and what proportion of these women develops complications. However, the cases described by Pillai et al. highlight the potential for lifethreatening infection and a lack of awareness of the need for IUD removal among some IUD user and health professionals. Current FSRH Guidance does not emphasise the need to advise patients about the importance of IUD remova when no longer required and about the potential risks of long-term IUD retention. We are gratefu to Dr Pillai and colleagues for drawing this to our attention and we shall ensure that recommendation to this effect is included in future updates of the Guidance on 'Contraception for Women Aged Over 40 Years' and 'Intrauterine Contraception

Case reports are a useful source of evidence where no other evidence exists. We would encourage others to report complications that are particularly rare, serious or associated with prolonged contraceptive use

Louise Melvin, MRCOG, MFSRH

Director, FSRH Clinical Effectiveness Unit

(CEU) and Consultant in Sexual and

Reproductive Health, Sandyford, Glasgow, UK.

E-mail:louise.melvin@ggc.scot.nhs.uk

\section{References}

Pillai M, Van der Venne M, Shefras J. Serious morbidity with long-term IUD retention [Letter]. J Fam morbidity with long-term IUD retention [ $L$

Fanuly of Sexul ane 2000; $35: 132$. Faculty of Sexual and Reproductive Healthcare Clinical Effectiveness Unit. FSRH Clinical Guidance (November 2007). Intrauterine contraception h.//Www.tpric.org.uk/admin/uploads/CEUGuidance Janutery 2009

\section{IUS producing a TAC}

I recently saw a very unusual patient in whom an intrauterine system (IUS) appeared to produce a trigeminal autonomic cephalgia (TAC).
The patient, a 39-year-old woman, was fortunate never to have had a headache until the events reported here. In early 2007, the patient started to complain of severe menorrhagia. Her periods were heavy and lasted 14 days, and necessitated the use of 15-20 sanitary pads a day. Tranexamic acid $1000 \mathrm{mg}$ qds was tried initially for 8 weeks but the heavy bleeding continued. Next a therapeutic trial of norethisterone $5 \mathrm{mg}$ tds was tried for many months resulting in a mild improvement. In desperation, the patient was referred to a gynaecologist who felt that the next step was to insert a levonorgestrel-releasing IUS. This was duly done. Within 6 hours of inserting the IUS the attacks started. All the patient's attacks (averaging 5-7 attacks/day) were similar. All were left sided and lasted 15-30 minutes. An attack started with pain to the side of the left eye that the patient described as unbearable, like the worst toothache ever. Associated with the pain was profuse tearing mainly from the left eye, although the pain was so bad the patient also cried with her right eye. Her palpabral fissure narrowed, her nose ran and her eye became pink. Her face felt strange and numb though painful. Touching her face, or brushing her hair or her teeth, did not trigger an attack. The attacks continued daily for 4 weeks until the patient came to see me.

As she entered the room, an attack started. Following the attack I removed the patient's IUS very easily and gave her a zolmitriptan nasal spray in case she had further attacks. I arranged to see her 7 days later, at which time she appeared to be a completely different person. She had suffered one further attack some 6 hours after the IUS was removed and so had used the nasal spray. After this her attacks had totally stopped. At that clinic visit, in order to help her menorrhagia, which still raged, I started the patient on norethisterone again. Eighteen months later she is still totally free of attacks, and although her bleeding is still very heavy, she is not prepared to even consider allowing me to reinsert an intrauterine device/system, with or without hormones. She says the pain was the worst pain she could ever imagine and as a result she would never, even for the purposes of research, have an IUS inserted again.

This woman appeared to develop a TAC, which approximated most closely to a cluster headache, though some attacks lasted only 15 minutes. It might be argued that it was not the IUS itself, but the hormone present in the IUS, which triggered the attacks, however this seems unlikely. The patient had already had very large doses of progestogen prior to IUS insertion with no ill effects and has also had large doses following IUS removal. The progesterone dose in the IUS is effective locally and is unlikely to have reached a high level after only 6 hours. Conversely, if the problem were the hormone in the IUS, its removal would be unlikely to cause the hormone level to decrease significantly in 6 hours.

In summary, on the face of it this would appear to be a simple case of a woman having an IUS inserted and developing a TAC, which was rapidly cured by removing the device. I would be delighted to discover if any of the Journal's readers have observed a similar case.

Susan L Lipscombe, $\mathrm{MBChB}$, MRCP Park Crescent New Surgery, Brighton, UK E-mail: suelipscombe1@ntlworld.com

\section{Informed consent for IUD fitting}

Perforation of the uterus is a rare complication of intrauterine device (IUD) fitting. It is quoted as occurring in up to 2 in 1000 IUD fittings. ${ }^{1}$ Risk factors for perforation include previous caesarean section $^{2}$ and postpartum insertion up to 6 months after delivery. ${ }^{3}$

Perforation may occur during the sounding of the uterus or the device itself may perforate the uterus. This can lead to the device being free in 